\title{
Frases nominales predicativas de orientación temporal*
}

\author{
ARMANDO MORA BUSTOS** \\ ALEJANDRA ITZEL ORTIZ VILLEGAS ${ }^{* * *}$
}

Recepción: 10 de febrero de 2020

Aprobación: 7 de junio de 2020

Forma de citar este artículo: Mora, A. \& Ortíz A. I. (2020). Frases nominales predicativas de orientación temporal. Cuadernos de Lingüística Hispánica, (36), 39-58.

doi $10.19053 / 0121053$ X.n36.2020.10673

* Artículo de investigación. Este trabajo hace parte del proyecto: Gramática descriptiva y tipológica de las lenguas de México: correlación entre niveles de análisis (Universidad Autónoma Metropolitana-Unidad Iztapalapa) y del proyecto interinstitucional: Grupo de Datos Lingüísticos, Universidad Autónoma Metropolitana (UAM), Instituto de Educación Media Superior (IEMS) y el Instituto Nacional de Antropología e Historia (INAH).

** Armando Mora Bustos es doctor en Lingüística por El Colegio de México (COLMEX), actualmente es profesor-investigador de la Universidad Autónoma Metropolitana, unidad Iztapalapa, México. Correo electrónico: amora@xanum.uam.mx @https://orcid. org/0000-0003-0743-4744

*** Alejandra Itzel Ortiz Villegas es candidata a doctora en Lingüística por la Universidad Nacional Autónoma de México, actualmente es docente-tutor-investigador del Instituto de Educación Media Superior de la Ciudad de México, México. Correo electrónico: aov_26@yahoo.com Đhttps://orcid.org/0000-0002-4107-4968 


\section{Resumen}

En este trabajo se plantea que los adjuntos expresados en una frase nominal de orientación temporal tienen una función predicativa. Las construcciones objeto de estudio son del tipo: se negaron a ser trasladados el miércoles. La descripción de estas frases nominales se fundamenta en el hecho de que semánticamente la frase nominal temporal es un predicado eventivo y sintácticamente esta unidad gramatical puede 0 no ser requerida por el verbo. El aporte de este trabajo se centra, por una parte, en la identificación de un argumento sintáctico y en la caracterización sintáctica y semántica de un adjunto; y por otra, en la justificación del carácter predicativo de los adjuntos. La forma como saturan sus argumentos los verbos y los adjuntos no es de la misma naturaleza sintáctica, pero sí, semántica. Los datos utilizados para ilustrar este hecho de lengua han sido retomados de diferentes fuentes.

Palabras clave: predicación; adjunto; argumento; frase temporal.

\section{Time-Oriented Predicative Nominal Sentences}

\section{Abstract}

In this work it is stated that the adjuncts expressed in a time-oriented nominal sentence have a predicative function. Constructions under study are of the type: they refused to be moved on Wednesday. The description of these nominal sentences is based on the fact that semantically the temporal nominal phrase is an eventive predicate and syntactically this grammatical unit may not be required by the verb. The contribution of this work focuses, on the one hand, on the identification of a syntactic reasoning and on the syntactic and semantic characterization of an adjunct; and on the other hand, on the justification of the predicative character of adjuncts. The way verbs and adjuncts saturate their reasoning is not of the same syntactic nature, but semantic. Data for illustrating this language fact have been taken up from different resources.

Keywords: predication, adjunct, reasoning, temporal sentence. 


\section{Phrases nominales prédicatives à orientation temporelle}

\section{Résumé}

Ce travail prétend démontrer que les éléments adjoints, exprimés dans une phrase nominale à orientation temporelle, ont une fonction prédicative. Les constructions objet d'étude sont du type: « ils ont refusé d)être transférés mercredi ». La description de ces phrases nominales est basée sur le fait que la phrase nominale temporelle est, du point de vue de la sémantique, un prédicat éventuel et que cette unité grammaticale peut ou non, du point de vue de la syntaxe, être rendue obligatoire par le verbe. La contribution de ce travail porte, d'une part, sur l'identification d'un argument syntaxique et sur la caractérisation syntaxique et sémantique d'un élément adjoint; et elle porte, d'autre part, sur la justification de son caractère prédicatif. La façon dont les verbes et les éléments adjoints saturent leurs arguments n'est pas de même nature syntaxique, mais elle est plutôt sémantique. Les données utilisées pour illustrer ce fait de langage proviennent de différentes sources.

Mots clés: prédication; élément adjoint ; argumentation ; phrase temporelle.

\section{Frases predictivas nominais de orientação temporária}

\section{Resumo}

Neste trabalho, afirma-se que os anexos expressos em uma frase nominal de orientação temporal têm uma função predicativa. Os edifícios em estudo são do tipo: eles se recusaram a ser transferidos na quarta-feira. A descrição dessas frases nominais é baseada no fato de que semanticamente a frase nominal temporal é um eventual predicado e, sintaticamente, esta unidade gramatical pode ou não ser requerida pelo verbo. A contribuição deste trabalho concentra-se, por um lado, na identificação de um argumento sintático e na caracterização sintática e semântica de um apego; e, por outro, na justificativa do caráter predicativo dos anexos. A maneira como verbos e adjuntos saturam seus argumentos não é da mesma natureza sintática, mas sim, semântica. Os dados usados para ilustrar esse fato da linguagem foram obtidos de diferentes fontes.

Palavras-chave: pregação; apego; argumento;frase temporal 


\section{Introducción}

Los adjuntos (adverbios o circunstanciales) han sido considerados como una serie de constituyentes que gramaticalmente tienen una función diferente a la de las relaciones gramaticales 0 argumentos sintácticos, dado que los primeros se conciben como modificadores no seleccionados por el predicado, aunque se manifiesten ciertas restricciones de aparición debido a distintas características tanto del predicado al que modifican como del significado del adjunto en sí (Real Academia Española [RAE], 2009). El objetivo de este trabajo es justificar la idea de que los adjuntos efectivamente tienen un comportamiento sintáctico particular y que semánticamente estas unidades gramaticales son susceptibles de predicar y en consecuencia de demandar su o sus argumentos. Particularmente, en este estudio se toman como unidad de análisis las frases nominales de orientación temporal, ilustradas de (1)-(3). La estructura de las frases nominales temporales es similar a la estructura de un FN prototípica, esto es, disponen de un núcleo y una serie de modificadores (artículos, demostrativos, cuantificadores, adjetivos, complementos prepositivos, cláusulas relativas). Los núcleos nominales de este tipo de frases refieren días (1a) y (1b), meses (1c), semanas (1d) y años (1e); temporales como momento (2a) y hora (2b), o bien, formas deícticas temporales como ayer (3a) o mañana (3b). El aporte de esta investigación se centra en profundizar la idea sobre la capacidad predicativa de estas unidades gramaticales.

1 a. La última vez caminé dos días en el desierto, dos noches con días completos.

b. Una laparoscopia te la hago el viernes; descansas sábado y domingo y ya el lunes te vas a la escuela.

c. Hubo muchas limitantes, nos costó ocho meses, ocho siete meses de trámite.

d. Llegó este cuate una semana antes y estuvo con nosotros viendo qué onda ahí en mercadotecnia.

e. Mi mamá lo que hizo fue no dejarnos, convivir con los demás y eso duró unos cinco años más o menos que fue cuando ocurrió lo del sismo.

2 a. Nos dijeron que los esperáramos un momento.

b. Una hora después encontramos en camino para llegar al cerro. 
3 a. Ayer se compraron todas las medicinas que nos pidieron.

b. Mañana vamos a ir por unos tacos de cochinita.

La clase de frases nominales temporales, como de (1) a (3), no son muy productivas; los núcleos de estas frases están limitados a un pequeño conjunto de sustantivos que refieren unidades de calendario. Las frases nominales de orientación temporal se ubican en cualquier posición dentro de la oración en la que aparecen, es decir, antes del verbo principal o después; es importante mencionar que en lenguas como el inglés algunos sentidos de locación y dirección también se expresan en este tipo de frases nominales (Larson, 1985).

\section{Metodología}

Esta investigación se realizó con ejemplos del habla de la Ciudad de México, estos fueron tomados de dos fuentes: la primera de ellas corresponde al "Proyecto para el Estudio Sociolingüístico del Español de España y de América” (PRESEEA); la segunda fuente es el "Corpus de Referencia del Español Actual" (CREA). Los datos que aquí se exponen representan diferentes tipos de contextos, a pesar de no tener la intención de realizar un estudio en términos cuantitativos, se ha tenido cuidado de mostrar la diversidad estructural en donde estas frases nominales con sentido temporal pueden aparecer. Como se mencionó anteriormente, la finalidad es mostrar el comportamiento de este tipo de frases tanto semántica como sintácticamente y argumentar a favor de que estos adjuntos son unidades predicativas y modificadoras del evento, lo anterior considerando la propuesta de la gramática de rol y referencia (Van Valin, 2005).

La estructura de este trabajo está ordenada de la siguiente manera: en un primer momento se revisa lo que se ha considerado como adjunto; posteriormente, se describen los rasgos que caracterizan a los adjuntos, tanto como unidades sintácticas como unidades semánticas; por último, se explica por qué debe considerarse el adjunto como una forma con función predicativa.

\section{Marco teórico}

En las últimas décadas, los estudios sobre la naturaleza gramatical de los adjuntos, adverbios o complementos circunstanciales en la lengua española han aumentado considerablemente; simplemente se deben revisar los trabajos de Bosque (1989), Bosque y Demonte (1999), Torner Castells (2005), Ibáñez (2012), Mora-Bustos (2012, 2014, 2019) y Mora-Bustos y García Zúñiga (2018), entre muchos otros. Los 
adverbios pertenecen a las categorías gramaticales más difusas, más amplias y más complicadas de caracterizar, sistematizar y clasificar. Por ejemplo, en (4) se presenta la distribución de algunos adverbios dentro de la frase adverbial y dentro de la oración, esto es, adverbios modificadores de verbos, (4a); adverbios modificadores de adjetivos, (4b); adverbios modificadores de otro adverbio, (4c); adverbios subcategorizados, (4d); adverbios atributivos o predicativos, (4e); adverbios introducidos por preposiciones, (4f); adverbios que se coordinan, (4g); adverbios que se pueden mover dentro de la oración, (4h); adverbios que no se pueden mover dentro de una oración, (4i); adverbios léxicos 0 con sentido funcional, (4j).

4 a. Los cantantes llegaron el lunes.

b. Es una mujer extraordinariamente bella.

c. No te puedo ver si estás allá lejos.

d. En esta ciudad se vive bien.

e. La niña canta alto.

f. Nos perdimos cuando veníamos por allá.

g. Los antibióticos actuaron rápida y eficazmente.

h. Solo compraron (solo) la bicicleta.

i. Tal vez vaya al cine (*tal vez).

j. Los atletas llegaron muy arriba.

Como se puede ver en los ejemplos de (4), las funciones gramaticales de los adverbios son diversas. Estas unidades léxicas, frasales y oracionales, como en (5), funcionan dentro de la estructura de la cláusula como adjuntos periféricos. En (5a) el adverbio demostrativo aquí indica el lugar donde trabajan los empleados, en (5b) alegremente indica la forma en la que María caminaba, en (5c) la frase nominal el lunes especifica el día en el que se presentará el examen, la frase prepositiva en la delegación (5d) ubica el espacio donde se realiza la lectura, el adverbio relativo introducido por la preposición en donde hace referencia a un lugar específico (5e). Lo anterior muestra que sin importar su posición dentro de la cláusula su estatus sintáctico es estrictamente periférico. 
5 a. Aquí todos tomamos mezcal.

b. Las mujeres con sus trajes típicos caminaban alegremente.

c. Nos han pedido que las escrituras las firmemos el lunes.

d. Nadie quiere atendernos en la delegación.

e. La idea es que vaya a la escuela en donde trabaja su papá.

En general, estas unidades gramaticales periféricas (5) han sido clasificadas a partir de diferentes criterios gramaticales, ya sean semánticos, distribucionales 0 morfológicos, este último rasgo fundamentado en la productividad. Estas clasificaciones se han ido incrementando de manera vertiginosa en los últimos años, tras la idea de que los adjuntos deben estudiarse a partir de su comportamiento gramatical más que de sus propiedades semánticas.

En este trabajo se parte de la idea de que todas las unidades gramaticales de (5) son adjuntos; dado que constituyen una clase natural, su función es la de modificar una de partes de la oración; de igual forma anclan o escenifican al o a los participantes del estado de cosas que está(n) expresando en la oración. Estos adjuntos forman una clase gramatical, pues tienen propiedades lingüísticas comunes, son sensibles a la sustitución, a la unión y al desplazamiento; a este respecto, no se olvide que debe haber afinidad 0 compatibilidad entre la unidad sustituidora con la sustituida, la del coordinante con el coordinado o la de la pregunta con la respuesta (Bosque \& Gutiérrez-Rexach, 2009). En el presente estudio se descarta la posición de que los adjuntos son unidades que se agregan a la oración opcionalmente, nótese que no hay equivalencia semántica o la simplificación es excesiva en el contraste realizado entre (6a) y (6b).

6 a. Todos los días trabajo en la casa de mi mamá.

b. Trabajo.

De acuerdo con este panorama, en este trabajo quedan fuera de estudio los complementos o los adjuntos que aparecen con verbos de movimiento, (7a); los adjuntos obligatorios (Mora-Bustos, 2019), (7b); los complementos de régimen prepositivo, (7c); los argumentos adjuntos (Jolly, 1993), (7d); los argumentos oblicuos del core (Belloro, 2007), (7e); los adverbios preposicionales (Alcina \& Blecua, 1994), (7f); y los modificadores adverbiales (Mora-Bustos \& García Zúñiga, 2018), (7g). Cada uno de estos 
tipos de adjunto tiene un comportamiento gramatical propio, en consecuencia, deben ser descritos y estudiados independientemente.

$7 \quad$ a. $\quad$ No pudieron llegar $\boldsymbol{a}$ la milpa.

b Los campesinos viven mal.

c. Nos tocó hablar una y otra vez con el delegado.

d. Pusieron el guajolote y el mole sobre la mesa.

e. $\quad$ A María le duele la cabeza.

f. Nuestros abuelos trabajaban duro dentro de la mina.

g. Allá arriba cantan los pajaritos.

Las frases nominales temporales (locativas y de dirección) tienen la misma estructura que la de una frase nominal simple. El grado de determinación es similar a lo que ocurre en una frase nominal argumental, al respecto, para el inglés, algunas frases nominales con sentidos locativos y de dirección han sido etiquetadas como 'Bare-NP adverb' (Larson, 1985).

Estructuralmente, los núcleos de estas frases también pueden aparecer modificados por una cláusula relativa (8a). Las funciones distribucionales de las frases nominales temporales adverbiales son similares a las que presentan las frases prepositivas $(8 \mathrm{~b}) 0$ las frases adverbiales (8c).

$8 \quad$ a. Ese sábado que secuestraron a los estudiantes, no salimos a ninguna parte.

b. Vendemos en las carreras cualquier día de la semana.

c. Convivían con los maestros todo el santo día.

Smith (1991), parala lengua inglesa, propone una clasificación de los modificadores temporales en función de sus condiciones de anclaje. Los adverbios temporales son clasificados como puede verse en (9); locativos (9a), durativos (9b) y frecuencia (9c).

9 a. Adverbios locativos: en la noche, ayer, el lunes, después de que María se fue, etc. 
b. Adverbios durativos: durante una hora, de 1:00 a las 3:00 p. m., etc.

c. Adverbios de frecuencia: a menudo, nunca, tres veces a la semana, etc.

En la Nueva gramática de la lengua española (RAE, 2009), los adverbios de localización junto con los de duración y los de frecuencia constituyen el grupo de los adverbios de tiempo; estos especifican el momento o el período en que tiene lugar un determinado estado de cosas. Entre los intervalos denotados por el evento y por el adjunto temporal se puede establecer una relación de coincidencia o simultaneidad. De acuerdo con esta gramática, los adjuntos temporales de los verbos citar, convocar, esperar, quedar y algunos otros más, poseen la capacidad de asociarse con eventos posteriores a los que estos predicados designan (10).

10 a. Citaron a los padres de familia el martes.

b. Quedaron de verse en la delegación la próxima semana.

En este contexto puede designar el día en el que se fija la cita el martes (10a), pero también aquel en el que ha de tener lugar la próxima semana (10b). Los adjuntos temporales de localización se pueden agrupar en tres clases, véase (11), esto con respecto a la manera en que se orientan deícticamente, es decir, dependiendo de su anclaje deíctico ayer/lunes (12a), anafórico dos semanas antes (12b) o variable en el plazo de tres días (12c).

11 a. Adjuntos temporales orientados en relación con el momento del habla (anclaje deíctico).

b. Adjuntos temporales orientados en relación con un punto temporal que no sea el momento del habla (anclaje anafórico).

c. Adjuntos temporales orientados en relación con cualquier punto temporal (anclaje variable).

12 a. Ayer/el lunes, María perdió la cita con el doctor.

b. Dos semanas antes/la víspera de la fiesta, salieron los peregrinos.

c. Llegará en el plazo de tres días. 
Como se ha visto, los adjuntos pueden ser, categorialmente, grupos adverbiales, preposicionales, nominales e incluso oraciones subordinadas; sin embargo, aquí nos ha interesado describir el comportamiento de los adjuntos constituidos por frases nominales de orientación temporal.

\section{Discusión}

Se ha considerado que los adjuntos, como no son requeridos, pueden ser omitidos y que, de hecho, esa omisión no afecta la gramaticalidad de la oración (RAE, 2009); no obstante, aquí se plantea que estos adjuntos estructurados como frases nominales de orientación temporal no se pueden omitir de la oración en la que aparecen y tienen alcance sobre el evento. Esto nos lleva a reflexionar sobre ciertos criterios tanto de los argumentos como de los adjuntos.

\section{Argumentos frente a adjuntos}

Los argumentos sintácticos están asociados de manera cercana con el significado léxico inherente del predicado, mientras que los adjuntos, no. Los verbos ponen restricciones de selección a los argumentos, pero no a los adjuntos (Kroeger, 2008). Los argumentos o relaciones gramaticales deben ser únicos dentro de la oración, pero los adjuntos pueden multiplicarse libremente, dentro la oración principal o de la oración subordinada. Los núcleos de una frase u oración potencialmente pueden ser modificados por un número ilimitado de adjuntos (Tallerman, 2009).

Dentro de esta concepción, pertenecen a esta categoría lingüística los adjuntos libres (13a), los adjuntos obligatorios (13b), y los complementos oblicuos (13c).

13 a. Todos los días con este niño todo el mundo batalla.

b. El señor no se manda solo.

c. La secretaria renunció a su trabajo.

Considérese que el carácter periférico de estas unidades no está relacionado con su opcionalidad u obligatoriedad, sino con el estatus que tienen con respecto a los requerimientos argumentales del verbo y a su pertinencia pragmática, que tiene incidencia sobre el anclaje de un evento en un espacio, tiempo y circunstancia determinados. 


\section{Comportamiento de los adjuntos}

Los adjuntos muestran un comportamiento diverso en términos de forma y distribución en la oración; tienen un entorno y un comportamiento sintáctico específico. Son formas que, mayoritariamente, no están subcategorizadas por el verbo y no pueden realizarse como relaciones gramaticales. Tienen la función de modificar las diferentes partes de la oración, se pueden mover a lo largo de la construcción en la que aparecen; además, dentro de una oración pueden aparecer tantos adjuntos como sean necesarios. Los argumentos dependen del core (centro), mientras que en la periferia se localizan los adjuntos (Van Valin \& LaPolla, 1997; Van Valin 2005).

Semánticamente, los adjuntos complementan y modifican el significado de la oración, el verbo, el nombre y otros adjuntos, incluidos los adjetivos. A partir de sus propiedades léxicas, existen numerosas clasificaciones que, en términos generales, los agrupan en un amplio repertorio de categorías y subcategorías. Las propiedades léxicas de los adjuntos condicionan ciertos comportamientos sintácticos, como la posición que ocupan dentro de la oración; de esta manera, de acuerdo con su significado, los adjuntos pueden distribuirse en cuatro zonas: dos preverbales (14a) y (14b) y dos posverbales (14c) y (14d), que están influidas por el orden de constituyentes.

14 a. Había un letrero que decía "dos mil niños, todos los días duermen en la calle".

b. Un día de estos me voy a escapar a San Cristóbal.

c. Me tardo diez minutos en bajar.

d. Yo llegué un sábado.

Este tipo de adjuntos, como se dijo, tiene carácter periférico; su comportamiento sintáctico es variable puesto que modifican diferentes elementos dentro de la cláusula. En futuras investigaciones se debe describir la relación que existe entre el movimiento de estos adjuntos con el orden de constituyentes; la pregunta sobre cuál es la motivación gramatical del movimiento de estos adjuntos aún está abierta. 


\section{Resultados}

\section{Adjunto como unidad predicativa}

Semánticamente, todos los argumentos reciben papel temático y algunos adjuntos o elementos no requeridos pueden asociarse con nociones que se relacionan con papeles temáticos: instrumentos, locaciones, entre otros (15). Los papeles temáticos solo se asignan a unidades argumentales requeridas por el verbo; esta asignación se realiza sobre relaciones gramaticales y sobre adjuntos.

15 a. Pedro cortó la pizza con un cuchillo a las ocho de la mañana en la mesa de juntas

b. $\quad \operatorname{cortar}(\mathrm{x}, \mathrm{y}, \mathrm{z}, \mathrm{u}, \mathrm{v})$

c. cortar (Juan, la pizza, con un cuchillo, a las ocho de la mañana, en la mesa de juntas)

La frase prepositiva con un cuchillo es un adjunto; se le asigna el papel semántico de instrumento. Lo mismo ocurre con los adjuntos: a las ocho de la mañana y en la mesa de juntas, que expresan contenidos de ubicación, tanto espacial como temporal. Bosque y Gutiérrez Rexach (2009) señalan que la estructura de un predicado especifica la naturaleza de sus argumentos, no la de los adjuntos, entonces se preguntan: ¿en dónde se debe mirar para saber algo sobre sus adjuntos? Este cuestionamiento se plantea bajo la consideración de si se asume una correlación estricta entre las propiedades de ser argumento y recibir papel temático, de ser así, señalan que se deberían tratar las frases nominales como argumentos, de forma que se llegaría a una estructura argumentaltemática extendida.

Estos mismos autores plantean que un análisis en el que se manifieste la opcionalidad en la aparición de los complementos circunstanciales forzaría postular entradas léxicas diferentes en función del número de circunstantes que aparecieran (Bosque \& Gutiérrez-Rexach, 2009). Este problema es denominado como el problema de la valencia variable de los predicados (Davidson, 1967). Dicho esto, el predicado cortar podría tener cuatro (16), tres (17) o dos argumentos (18).

16 a. Juan cortó la pizza con un cuchillo a las ocho de la mañana

b. $\quad \operatorname{cortar}(\mathrm{x}, \mathrm{y}, \mathrm{z}, \mathrm{u})$

c. $\quad$ cortar (Juan, la pizza, con un cuchillo, a las ocho de la mañana) 
17
a. Juan cortó la pizza con un cuchillo
b. $\quad \operatorname{cortar}(\mathrm{x}, \mathrm{y}, \mathrm{z})$
c. $\quad$ cortar (Juan, la pizza, con un cuchillo)

18 a. Juan cortó la pizza

b. $\quad \operatorname{cortar}(\mathrm{x}, \mathrm{y})$

c. $\quad$ cortar (Juan, la pizza)

Para Davidson (1967), estos complementos circunstanciales no son considerados como argumentos del verbo principal, sino como predicados monádicos independientes, es decir, el único argumento de estos predicados es un argumento de evento o argumento eventivo.

\section{Frase nominal de orientación temporal como argumento eventivo}

Las frases nominales de orientación temporal pueden considerarse como propiedades de un evento; de hecho, Davidson (1967) considera los complementos circunstanciales 0 adjuntos como predicados de eventos. De esa manera, el complemento circunstancial indica una propiedad del suceso o evento expresado en la oración, esto es, a las cinco de la tarde (19a) y el veinticinco de mayo (20a) son propiedades del evento llegar, como puede verse en (19b) o de los eventos terminar y regresar de (20b).

19 a. Juan llegó a las cinco de la tarde

b. A las cinco de la tarde (e).

20 a. Ya terminé y regreso el veinticinco de mayo

b. $\quad$ El veinticinco de mayo (e).

En específico, el sintagma prepositivo o la frase nominal temporal expresa una propiedad temporal de dicho evento: la llegada de Juan tuvo lugar a las cinco de la tarde, el evento de que una primera persona terminó algo y el regreso de esta ocurrirá el veinticinco de mayo. Esto corresponderá, por lo tanto, a un predicado saturable por un argumento que se asocia con sucesos o eventos, es decir, el argumento eventivo (e). Así, 
la oración de (19a) debe ser reducible semánticamente a una paráfrasis como "hay un evento $e$ tal que Juan llegó en $e$ y $e$ tuvo lugar a las cinco de la tarde"; por su parte (20a) debe ser reducible semánticamente a la paráfrasis "hay un evento $e$ tal que yo terminé y regreso en $e$ y $e$ tuvo lugar el veinticinco de mayo".

La cuestión prioritaria es entonces determinar cuál es el estatus de este argumento eventivo y cómo es posible asociar los argumentos de individuo y el argumento eventivo. Al respecto, se debe tener presente lo que Jespersen (1924) expone ante el siguiente paralelismo: la relación semántica que se da entre los adjetivos y los sustantivos a los que estos modifican (estudio pormenorizado) es análoga a la que existe entre los adverbios de manera y los verbos sobre los que estos inciden (estudiar pormenorizadamente). Dicho así, el concepto de complemento circunstancial no nos ayuda a establecer este paralelismo. El adjetivo pormenorizado selecciona y, por lo tanto, restringe semánticamente ciertos tipos de entidades entre las que está estudio. En el mismo sentido, el adverbio pormenorizadamente selecciona y restringe semánticamente la categoría sobre la que incide, en este caso el verbo estudiar, sin embargo, para poder hacerlo ha de interpretarse como un predicado. En el caso de los adjetivos, el concepto de "predicación generalizada" permite entender que un modificador puede ser a la vez un predicado. Dadas estas características, es necesario extender a los adverbios alguna variante de este concepto, si se quiere mantener el paralelismo entre adjetivos y adverbios (Bosque \& Gutiérrez-Rexach, 2009).

Ahora bien, se debe recordar que la noción de predicación es semántica, hace referencia a una condición relativa al significado de las expresiones. El concepto de predicación -análisis lógico y filosófico- (Kalish \& Montague, 1965; Deaño, 1978) no se restringe a la predicación oracional, se puede considerar como una estructura predicativa cualquier relación de saturación entre una función proposicional y su argumento (Bosque \& Gutierrez-Rexach, 2009). Ya Bello (1984) y Bach (1968) propusieron esta situación en el sentido de que los nombres y los adjetivos son predicados.

Davidson (1967) también considera que los verbos no solo expresan relaciones entre sus participantes, sino que asocian dichos participantes con un evento, así todos los verbos poseen un argumento eventivo. De hecho, un verbo que ha sido caracterizado como monádico pasa a tener dos argumentos: el argumento que hace referencia a entidades o individuos y un argumento eventivo. Así, los argumentos sintácticos de un verbo como recortar de (21) se saturan por rección, a diferencia de esto, su argumento eventivo no se satura de esta forma, ya que no representa a un participante.

a. El otro día Luis recortó el árbol con unas tijeras pequeñas. 
b. El otro día (e)

c. El otro día ((e) recortar $(\arg 1, \arg 2)))$

El argumento eventivo de un verbo se iguala o se identifica con la posición del argumento eventivo de los predicados de eventos, es decir, los modificadores 0 complementos circunstanciales; esto indica que tanto el modificador como el verbo se refieren al mismo evento.

\section{Frases nominales de orientación temporal como predicados de un lugar}

Van Valin y La Polla (1997) y Van Valin (2005) mencionan que los adverbios no están restringidos a la periferia y pueden modificar diferentes partes de la cláusula. Semánticamente, de acuerdo con el planteamiento de Jackendoff (1972), se consideran los adjuntos 0 adverbios como predicados de un lugar (one-place predicates) que toman la estructura lógica 0 una subparte de la estructura lógica como su argumento. Las frases nominales adverbiales periféricas, como el sábado (22a) y ayer (23a), toman la estructura lógica de la oración como su argumento. Los adverbios temporales toman toda la estructura lógica como su argumento.

22 a. Pedro horneó un pastel el sábado

b. el sábado' ([do' (Pedro, Ø)] CAUSE [BECOME horneó' (pastel)])

23 a. Ayer Cristina corrió al parque.

b. ayer' (do' (Cristina, [run' (Chris)]) \& INGR be-at' (parque, Cristina))

En caso de que aparezcan múltiples adjuntos, estos deben ser ubicados con respecto al predicado que se encuentre en la posición más alta de la estructura lógica, como en (24).

24 a. El sábado Pedro horneó un pastel en la cocina.

b. el sábado> (be-in> (cocina, [[do> (Pedro, Ø)] CAUSE [BECOME horneós(pastel)]])) 
Junto con los adverbios temporales, los adverbios epistémicos (25) y los evidenciales (26) también tienen alcance sobre la cláusula.

25 a. Evidentemente, Pedro horneó un pastel en la cocina el sábado.

b. evidentemente> (el sábado> (be-in> (cocina, [[do> (Pedro, Ø)] CAUSE [BECOME horneó (pastel)]])))

26 a. Probablemente, mañana Pedro horneará un pastel.

b. probablemente> (mañana> $([\mathrm{do}>($ Pedro, $\varnothing)]$ CAUSE [BECOME horneór (pastel)]))

La posición de las frases nominales temporales dentro de la estructura lógica de una oración compleja se ubica como predicados en la parte más alta de esa estructura lógica, como puede verse en (27).

27 a. Esta mañana Antonio salió a Puebla y mañana llegará a Oaxaca.

b. [esta mañana' [do' (Antonioi,[salió' (Antonioi, Ø)]) \& BECOME NOT be-in' (Puebla, Antonioi]] and' [mañana (BECOME be-in' (Oaxaca, proi))]

En suma, se puede decir que hay tres clases de adjuntos no argumentales: a) los adjuntos expresados en frases prepositivas, b) los adjuntos expresados en frases nominales y c) los adjuntos léxicos, es decir, los adverbios. Las frases prepositivas modifican el núcleo verbal cuando estos expresan locación o rasgos temporales de los estados de cosas que se codifican en este núcleo verbal (28a). Las frases nominales modifican el núcleo verbal y especifican la referencia temporal de ese estado de cosas (28b). Por último, los adverbios léxicos modifican también el núcleo verbal y su(s) argumento(s); es decir, los adverbios temporales, como ayer, también ocurren en la periferia (28c). Igualmente, los adjuntos léxicos pueden modificar las diferentes partes de la cláusula; los adverbios aspectuales modifican el núcleo (28d), los adverbios de velocidad y manera modifican el verbo más su(s) argumento(s) (28e); los adverbios epistémicos y evidenciales modifican la cláusula (28f). En consecuencia, las unidades periféricas que expresan estos sentidos son modificadoras del núcleo verbal más su(s) argumento(s).

28 a. Todos se reunieron en el estadio. 
b. La semana pasada hice el examen.

c. Ayer los niños se fueron de paseo.

d. Vive todavía.

e. La señora bailaba bien .

f. Efectivamente mañana saldremos temprano.

En cuanto a la estructura lógica, las frases prepositivas son adjuntos periféricos que por definición siempre son predicativos, ya que estos no marcan argumentos del verbo. Estos argumentos periféricos modifican el verbo y su(s) argumento(s) como un todo, toman la estructura lógica del verbo de la cláusula como uno de sus argumentos, como en (29) y (30).

29 a. Pedro escribió una carta en la escuela.

b. be-in' (escuela, [[do' (Pedro, Ø)] CAUSE [BECOME escribió' (carta)]])

30 a. Pedro escribió una carta después de trabajar.

b. be-after $>($ trabajo, $[[$ do $>($ Pedro, $\varnothing)]$ CAUSE [BECOME escribió' (carta)]])

Semánticamente, las frases nominales temporales, las frases prepositivas y los adverbios léxicos están representados como los predicados más altos dentro de la estructura lógica. En (31), la frase nominal el lunes es una proposición predicativa que toma la cláusula de relativo y toda la estructura lógica que se genera para Ana vio Arturo como sus argumentos. En cuanto a las frases nominales Ana y Arturo están unidas en la posición de dos argumentos dentro de la estructura lógica generada para ver, mientras que la cláusula de relativo está unida en una posición argumental en la estructura lógica como se ve representado en (31b).

31 a. El lunes que salimos de vacaciones, Ana vio a Arturo.

b. el lunes> (vaciones' (salir' (nosostros))), [ver> (Ana, Arturo)]) 
En síntesis, estas unidades gramaticales, adjuntos periféricos, tienen la posibilidad de modificar distintas partes dentro de la cláusula. Las frases nominales de orientación temporal toman como argumento toda la estructura lógica, por ello, se representan en la parte más alta de la misma.

\section{Conclusiones}

En este artículo se ha desarrollado la idea de que los adjuntos, las frases nominales de orientación temporal, son unidades gramaticales susceptibles de generar o demandar su 0 sus argumentos; es decir, son unidades que de manera similar a los adverbios y adjetivos predican el evento o los eventos sobre los que tienen dominio o alcance. Nótese que esta idea no es nueva, ya ha sido reconocida y planteada por algunos lingüistas; la novedad y el aporte de este trabajo se ha centrado en describir y justificar a través de una forma gramatical específica, la plausibilidad de que los adjuntos, semánticamente, son en realidad unidades predicativas.

En este trabajo se ha sustentado que las frases nominales de orientación temporal, al formar parte de la clase de los adjuntos, no son requeridas sintácticamente por el verbo; sin embargo, se ha manifestado que son necesarias semánticamente para completar el significado de la oración de la que forman parte, es decir, tienen función predicativa; los núcleos de estas frases nominales hacen referencia, sobre todo, a unidades de calendario.

Estos adjuntos no argumentales expresados por frases prepositivas, frases nominales o adverbios se agrupan dependiendo de su anclaje, ya sea deíctico, anafórico 0 variable; de igual modo, los adjuntos periféricos son considerados como predicados monádicos, los cuales tienen como único argumento un evento. Por último, las frases nominales con orientación temporal, al ser modificadores eventivos, se encuentran en la posición más alta de la estructura lógica de la que forman parte.

Quedan pendientes para estudios posteriores algunos cuestionamientos que deben ser resueltos, como por ejemplo: itodos los adjuntos tienen la misma capacidad semántica de predicar?, icómo se correlacionan y coocurren estos adjuntos con carácter predicativo en los niveles sintáctico y semántico? Las respuestas a estas preguntas simplemente mostrarán que los fenómenos gramaticales de una lengua, muchas veces, no pueden ser explicados a partir de un solo nivel de análisis, sino que se deben considerar las correlaciones que se presentan entre estos. 


\section{Referencias}

Alcina, J. \& Blecua, J. (1994). Gramática española. Barcelona: Ariel.

Bach, E. (1968). Nouns and noun phrases. En E. Bach \& R. Harms (eds.), Universals in linguistics theory (pp. 90-122). Nueva York: Holt, Rinehard.

Bello, A. (1984). Gramática de la lengua española. Madrid: EDAF.

Belloro, V. (2007). Spanish clitics doubling. A study of the syntax-pragmatic interface. (Tesis de doctorado). Universidad Estatal de Nueva York en Búfalo.

Bosque, I. (1989). Las categorías gramaticales. Relaciones y diferencias. Madrid: Síntesis.

Bosque, I. \& Demonte, V. (Eds.). (1999). Gramática descriptiva de la lengua española, (vol. 3). Madrid: Espasa.

Bosque, I. \& Gutiérrez-Rexach, J. (2009). Fundamentos de sintaxis formal. Madrid: Akal.

Davidson, D. (1967). The logical form of action sentences. In D. Davidson, Essays on actions and events (pp. 105-148). 0xford: Oxford University Press. https://doi.org/10.1093/0199246 270.003 .0006

Deaño, A. (1978). Introducción a la lógica formal. Madrid: Alianza.

Ibáñez, S. (2012). Argumentos, adjuntos y frases preposicionales en español. En Mairal, R., Guerrero, L. \& González, C. (eds.), El funcionalismo en la teoría lingüística: la gramática del papel y la referencia (pp. 187-202). Madrid: Akal.

Jackendoff, R. (1972). Semantic interpretation in generative grammar. Cambridge: MIT Press.

Jespersen, 0. (1924). La filosofía de la gramática. Barcelona: Anagrama.

Jolly, J. A. (1993). Preposition assignment in English. In R. D. Jr. Van Valin (ed.), Advance in role and reference grammar (pp. 275-310). Amsterdam-Philadelphia: Benjamins. https:// doi.org/10.1075/cilt.82.08jol

Kalish, D. \& Montague, R. (1965). Logic. Techniques of formal reasoning. New York: Harcourt Brace.

Kroeger, P. (2008). Analyzing syntax. A lexical-functional approach. Cambridge: Cambridge University Press.

Larson, R. (1985). Bare-NP Adverbs. Linguistic Inquiry, (16), 595-621. 
Mora-Bustos, A. (2012). Los adjuntos periféricos. En R. Mairal, L. Guerrero \& C. González (eds.), El funcionalismo en la teoría lingüística: la gramática del papel y la referencia (pp. 202-224). Madrid: Akal.

Mora-Bustos, A. (2014). Verbos intransitivos y adjuntos. En P. Butragueño \& L. Orozco (eds.), Argumentos cualitativos y cuantitativos en sociolingüística: Segundo coloquio de cambio y variación lingüística (pp. 567-594). México: COLMEX. https://doi. org/10.2307/j.ctv6jmww1.24

Mora-Bustos, A. (2019). Adjuntos obligatorios o de régimen verbal. En N. Palacios (ed.), Voces de la lingüística mexicana contemporánea (pp. 205-234). México: COLMEX. https://doi. org/10.2307/j.ctvsf1pxh.10

Mora-Bustos, A. \& García Zúñiga, A. (2018). Modificadores adverbiales. En S. Bogard (ed.), Sentido y gramática en español (pp. 205-233). México: COLMEX.

Real Academia Española -RAE-. (2009). Nueva gramática de la lengua española. Madrid: Espasa.

Smith, C.S. (1991). The parameter of aspect. Dordrecht: Kluwer Academic Publishers. https:// doi.org/10.1007/978-94-015-7911-7

Tallerman, M. (2009). Understanding Syntax. London: Hodder Arnold.

Torner Castells, S. (2005). Aspectos de la semántica de los adverbios de modo en español. Barcelona: Unisersitat Pompeu Fabra.

Van Valin, R. (2005). Exploring the syntax and semantic interface. Cambridge: Cambridge University Press. https://doi.org/10.1017/CB09780511610578

Van Valin, R. \& R. LaPolla. (1997). Syntax. Structure, meaning, and function. Cambridge: Cambridge University Press. https://doi.org/10.1017/CB09781139166799 